immunoglobulin and Hepatitis B vaccine. All newborns had negative $\mathrm{HBsAg}$ on immediate post-birth.

Conclusion Generally, Hepatitis B patients had an average age from 20 to 30 years. The first appointment on specialised services was mainly on the second trimester, probably due to delays on public health dispensation. Hepatitis B vertical transmission prophylaxis has been done on $90 \%$ of the pregnant women - other cases may be lacking pertinent annotations. This evaluation needs to be carried on in order to minimise horizontal and vertical transmissions. Due to public dispensation mismanagement, viral load quantifications on Hepatitis B only started last year.

\section{P3.183 PREVALENCE AND FACTORS ASSOCIATED WITH SYPHILIS IN PREGNANT AND NON-PREGNANT WOMEN ATTENDED IN 24 MONTHS IN A UNIVERSITY HOSPITAL}

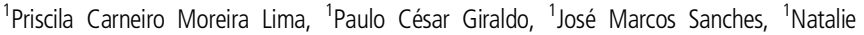
Rios Almeida, ${ }^{2}$ Mauro Romero Leal Passos, ${ }^{1}$ Rose Luce Gomes do Amaral. ${ }^{1}$ Unicamp, Campinas - SP, Brazil; ${ }^{2}$ Universidade Federal Fluminense, Niterói - RJ, Brazil

\subsection{6/sextrans-2017-053264.418}

Introduction It is estimated that 1.8 million pregnant women worldwide are infected with syphilis and less than $10 \%$ are diagnosed and treated. In Brazil, about 30 thousand cases/year with progressive growth are related to social inequalities and lost opportunities of diagnosis and prevention. Considering the importance of a faster detection of this disease, an analysis is necessary because such data could contribute to the development of new syphilis control strategies in this population.

Methods This retrospective case-control study included 170 women (34 cases and 136 controls), where the presence of VDRL and TPHA have defined as a case. The study was based on analyses of medical records of women admitted to the Women's Hospital Prof.Dr.José Aristodemo Pinotti/Unicamp-São Paulo-Brazil, matched by age over a period of 24 months. Statistical analysis was performed using chi-square and Fisher's exact tests. For the quantitative variables, descriptive measures were obtained and to verify a significant difference between the mean values, a Mann-Whitney test was used.

Results The prevalence was $1.04 \%$ and the mean age was 27.5a. $58.8 \%$ of women cases were white, $52.9 \%$ with a fundamental education and were in the 3.24 gestation (or 6.77). $70.6 \%$ of the controls were white with $45.6 \%$ average education and were at 2.27 gestation. In women with syphilis, the average number of sexual partners was 7 , the beginning of the sexual activity was $15.3 \mathrm{a}, 29.5 \%$ were drug users (or 0.0001 ), and $35.3 \%$ had a history of STDs. Controls had an average of 2.6 sexual partners, sex at $17.1 \mathrm{a}, 11.1 \%$ used drugs and $7.4 \%$ had a history of STDs. The cases had 6.2 consultations $(p=0.0664)$. Half of the partners were not treated. There was 1 neonatal death. Of the 34 women diagnosed with syphilis, only 5 achieved follow-up and cure.

Conclusion A high prevalence of syphilis was identified, which was associated with white, young and multiparous women. Socio-demographic vulnerability and difficulty in diagnosis seem to influence the disease, it is noteworthy that only 1 in 7 women obtained a cure.

\section{P3.184 TEMPORAL EVOLUTION OF RESISTANCE RATES AMONG CLINICAL ISOLATES OF NEISSERIA GONORRHOEAE FROM SÃO PAULO, BRAZIL}

${ }^{1}$ Rafael Affini Martiins, ${ }^{2}$ Roberto José Carvalho Da Silva, ${ }^{1}$ Dandara Cassu-Corsi, Carolina Silva Nodari'; ' $R$ Roberta Alessandra Lima Bocalon, ${ }^{1}$ Rodrigo Cayô, ${ }^{1}$ André Mario Doi, ${ }^{1}$ Ana Cristina Gales, ${ }^{1}$ Antonio Carlos Campos Pignatari. 1 Laboratório Especial de Microbiologia Clínica - LEMC/ALERTA, São Paulo - SP, Brazil; ${ }^{2}$ Centro de Referência e Tratamento de Doenças Sexualmente Transmissiveis - CRT/AIDS Santa Cruz, São Paulo - SP, Brazil

\subsection{6/sextrans-2017-053264.419}

Introduction The emergence of antimicrobial resistance among $N$. gonorrhoeae isolates is a major concern worldwide. Although quinolones and macrolides are still recommended for empirical treatment of urethritis according to our national guidelines.The objective of this study was to evaluate the antimicrobial susceptibility profile of $N$. gonorrheae recovered from 2003 to 2015 from outpatients assisted at the Centro de Referência e Treinamento DST/AIDS-CRT Santa Cruz, São Paulo - SP.

Methods The identification was carried out by MALDI-TOF MS. The minimal inhibitory concentrations (MIC) for penicillin, ceftriaxone, ciprofloxacin and azithromycin were determined by agar dilution method and interpreted according to CLSI (2016) clinical breakpoints, except for azithromycin, which was interpreted using EUCAST (2016). The genetic relationship of isolates presenting reduced susceptibility to ciprofloxacin was evaluated by ERIC-PCR. Hydrolysis rates towards ceftazidime and cefotaxime were evaluated by mass spectrometry.

Results Among the 125 n. gonorrhoeae recovered, reduced susceptibilities to penicillin, ciprofloxacin, and azithromycin were observed for $89.6 \%(112 / 125), 22.3 \%$ (21/94), and $26.4 \%$ $(33 / 125)$ of the isolates. Only one isolate was resistant to ceftriaxone, with MIC of $0.5 \mu \mathrm{g} / \mathrm{mL}$. Reduced susceptibilities to penicillin, ciprofloxacin and azithromycin were already observed in 2003, and increased over the years, while resistance to ceftriaxone was only observed in 2006. The ceftriaxone-resistant isolate did not present detectable hydrolysis for ceftazidime and cefotaxime, suggesting that a no enzymatic mechanism was involved.

Conclusion Our data corroborates with other international series and pose in question the recommended syndromic treatment with quinolones and azithromycin. Our result suggests that ceftriaxone still remains a valuable therapeutic option for the empirical treatment of gonococcal infections in Brazil. Further analysis will be performed in order to better characterise the genetic relationship and the resistance mechanisms involved.

\section{P3.185 ADOLESCENTS WITH HPV: THE PROFILE OF YOUNG PEOPLE ATTENDING A STI AND HIV REFERENCE CENTRE IN SALVADOR/BAHIA}

${ }^{1}$ Raiza Trindade, ${ }^{2}$ Eveline Xavier-Souza, ${ }^{2}$ Maiara Timbó, ${ }^{1}$ Krysna Lessa, ${ }^{1}$ Camila Souza, ${ }^{3}$ Ana Gabriela Travassos. 1 Universidade do Estado da Bahia, Salvador - BA, Brazil; ${ }^{2}$ Universidade Federal da Bahia, Salvador - BA, Brazil; ${ }^{3}$ Centro Estadual Especializado Em Diagnóstico, Assistência e Pesquisa (CEDAP), Salvador - BA, Brazil

\subsection{6/sextrans-2017-053264.420}

Introduction The adolescence is a period marked by intense vulnerability, with a high prevalence of HPV, mostly of 